\title{
A Convenient Synthesis of 8-Alkyl-2' (or 3')-azido (or amino)-2' (or 3')-deoxyadenosine as Diverse Synthetic Precursors of Cyclic Adenosine Diphosphate Ribose (cADPR)
}

\author{
Beom-Tae Kim, Bo-Seung Kim, ${ }^{\dagger}$ Chy-Hyoung Han, Kwang-Joong O, Sun-Ja Kim, \\ Jae-Chul Chun, Jin-Ho Lee, ${ }^{+}$Sung-Eun Kim, ${ }^{+}$and Ki-Jun Hwang ${ }^{*}$ \\ Department of Chemistry, "Department of Bioactive Material Sciences, College of Natural Science, \\ "Division of Applied Biotechnologv, College of Agriculture and Life Science, Research Center of Bioactive Materials, \\ Chonbutk National University, Chonju 561-756, Korea. E-mail: kijun@chonbuk.ac.kr \\ Received April 17, 2006
}

\begin{abstract}
$\Lambda$ s key nucleoside intermediates for the preparation of cyclic adenosine diphosphate ribose (c $\Lambda D P R, 1)$ analogues, 8-alkyl-2' (or 3')-azido(or amino)-adenosine derivatives (16-19) were successfully prepared by alkylating selectively protected adenosine derivatives $(12,13)$ via $\mathrm{Pd}(0)$ catalyzed cross-coupling reaction with tetraalkyltin reagents, followed by the sugar modification of these 8-alkyl-adenosine derivatives according to our precedent procedure. Compared to other precedent procedures, our 8-alkylation methodology using selectively TBDMS-protected 8-alkyl adenosine derivatives as starting materials will be utilized very conveniently to prepare highly functionalized adenosine analogues, which will be serve as key intermediates for the $c \Lambda D P R$.
\end{abstract}

Key Words : Cyclic adenosine diphosphate ribose (cADPR), 8-Alkyl-2' (or 3')-azido (or amino)-2' (or 3')deoxyadenosine

\section{Introduction}

Cyclic adenosine diphosphate ribose (CADPR, 1), a naturally occurring cyclic metabolite of $\mathrm{NAD}^{\prime}$, has been known to play an important role as a $\mathrm{Ca}^{21}$-mobilizing second messenger in various cellular events, such as glucosedependent insulin secretion in pancreatic $\beta$-cells, proliferation of human T-lymphocytes, arrhythmogenic oscillations of intracellular $\mathrm{Ca}^{21}$ in cardiac muscles. ${ }^{2}$ However, since the understanding of the role of $\mathrm{CADPR}$ in $\mathrm{Ca}^{2}$ signaling network is only just emerging, there have been continuous needs for the preparation of cADPR analogues having diverse structural modification to investigate the mechanism of cADPR-mediated signaling pathways in diverse cell systems and the therapeutic potentials of those compounds. ${ }^{3}$ The structural modifications, mostly occurring on the adenine or ribose sugar moieties of $\mathrm{CADPR}$, give rise to remarkable changes in their activity profiles; namely, showing considerable change in agonistic activity $(\mathbf{2}, \mathbf{5}, \mathbf{6})$, or showing no activity $(3,4)$, or even reversed activity (7-9) (Figure 1) ${ }^{3 d}$ Particularly, the cADPR analogues showing antagonistic activity in vertebrate cell systems have common and characteristic structural feature, i.e. the substitution at 8position of purine ring with diverse substituents, such as amino group (7), methoxy (8), bromine (9). ${ }^{4}$ This indicates that a substituent at 8-position of purine ring seems to be essential for antagonistic activity, even though this observation is based on the limited number of cADPR analogues. By considering the remarkable reversion of activity by chemical modifications on sugar or purine moieties of CADPR molecule and not many analogues found on our literature survey, we determined to prepare another type of cADPR analogues having chemical modifications on both of purine and sugar moieties.

As shown in Figure 1, the general synthetic strategy to prepare CADPR analogues includes intracyclization of $\mathrm{NAD}^{\prime}$ analogues, which in turn should be prepared from the elaborately pre-modified nucleosides. Therefore, the synthetic priority should be given to the step to develop new and diverse nucleosides..$^{5}$ In the meantime, we have already reported the convenient synthetic route to prepare sugarmodified adenosine derivatives having an azido group and an amine group at $2^{\prime}$ (or $3^{\prime}$ )-position of ribose moiety as key precursors to $\mathrm{NAD}^{1}$ derivatives. ${ }^{6} \mathrm{So}$, we decided to synthesize adenosine derivatives on which the chemical modifications occur at both of sugar and purine moiety, i.e. 8-alkyl-2' (or 3')-azido (or amino)-2' (or $3^{\prime}$ )-deoxyadenosine adenosine derivatives (16-19, in Scheme 1). This synthetic work would make it possible to extend the chemical diversity of the adenosine derivatives in hand, and could ultimately contribute to scrutinize structure-activity relationship of cADPR derivatives, which would be prepared from these nucleosides. In this paper, our synthetic efforts for sugar/ base-modified adenosine derivatives utilizing our previous synthetic methodology will be reported.

\section{Results and Discussion}

The precedent literature revealed that the introduction of alkyl substituents to 8-position of adenine moiety has been conventionally accomplished through halogenation followed by nucleophilic displacement, ${ }^{7}$ or lithiation of the 8-position followed by reaction with suitable electrophiles, ${ }^{8}$ or palladium $(0)$ catalyzed cross-coupling reaction with diverse organotin reagents. ${ }^{9}$ After scrutinizing the literatures, the last methodology is much likely to be promising over others in 
<smiles>[R]c1nc2c(N)ncnc2n1C1OC(COP(=O)(O)OP(=O)([O-])OCC2OC([n+]3cccc(C(N)=O)c3)C(O)C2O)C([R])C1[R]</smiles>

$\mathrm{NAD}^{+}$analogues
(1) Phosphorylated

(2) Coupled with NMN

NMN: Nicotinamide 5 -mononucleotide<smiles>[R]c1nc2c(N)ncnc2n1C1OC(CO)C([R])C1[R]</smiles>

Nucleosides

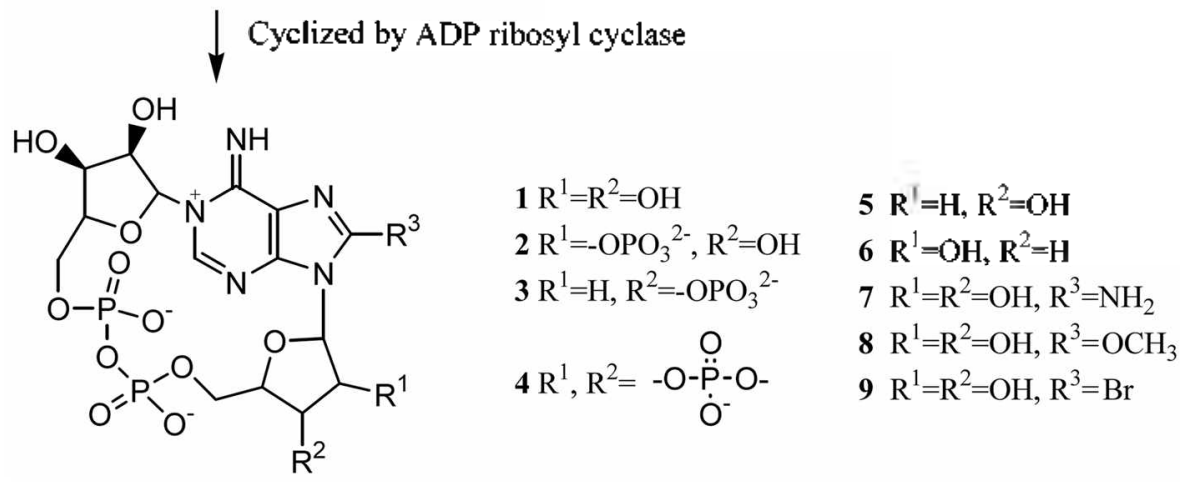

cADPR analogues

\section{Figure 1}

temms of yields and handling reactions, so it was utilized for the preparation of 8-alkyl adenosine derivatives.

8-Bromoadenosine derivatives $(12,13)$, the precursors for the 8-alkylation reaction, are easily obtained from selectively TBDMS-protected adenosine derivatives $(\mathbf{1 0}, \mathbf{1 1})^{6}$ with usual bromination condition ( $\mathrm{Br}_{2}, 1 \mathrm{M}$ acetate buffer (pH 4.3)-MeOH, rt) in high yields (>90\%, Scheme 1). For the successful 8-alkylation step, the optimum reaction condition was obtained by varying the concentrations of palladium $(0)$ catalyst $\left[\mathrm{Pd}\left(\mathrm{PPh}_{3}\right)_{4}\right]$ and tetraalkyltin (alky] $=$ methyl, ethyl, and vinyl), and range of temperature (shown in Table 1). The high reaction temperature around $100^{\circ} \mathrm{C}$ is required, while the reactions occurred very slowly or not at all at low reaction temperature $\left(30-80^{\circ} \mathrm{C}\right) .^{9}$ Under the applied reaction condition, the 8-alkylated adenosine derivatives having selectively protected sugar (14a-c and 15a-c) were successfully prepared in acceptable yields (70$90 \%$, Table 1).

With those compounds in hand, we moved on to prepare our target compounds, 8-alkyl-2' (or 3')-azido (or amino)-2' (or 3')-deoxyadenosine derivatives (16-19), simply by modifying the sugar moiety according to our already reported procedure. ${ }^{6}$ That is, the initial step was the conversion of sugar hydroxyl group at the 2' (and 3')-position of 14 (and 15) to azido group via arbino- or xylofuranosyl nucleosides, which provide the selectively TBDMS-protected 2' (or 3')azido-2' (or 3')-deoxyadenosine derivatives in good yields in each step (data not shown). From these compounds, the 2' (or 3')-azido compounds (16 and 17 ) were directly prepared with no incidence by the simple desilylation reaction condition $\left(\mathrm{NH}_{4} \mathrm{~F} / \mathrm{MeOH}\right.$ at $\left.60^{\circ} \mathrm{C}\right) .^{10}$ The desilylation step is apparently occurred in two-steps on the analysis of TLC, and so the selective desilylation of our particular compounds is possible upon needed. The next efforts were the reduction of azido group to amino group to prepare the 2' (or 3')-amino compounds (18 and 19). 8-Methyl (and ethyl)-2' (or 3')aminoadenosine derivatives $(18 a, 18 b$ and $19 a, 19 b)$ were easily obtained with the conventional hydrogenation condition $\left(\mathrm{H}_{2}, \mathrm{Pd} / \mathrm{C}, \mathrm{rt}\right)$ and subsequent desilylation. For the preparation of 8-vinyl-2' (or 3')-aminoadenosine derivatives $(18 \mathrm{c}$ and $19 \mathrm{c})$, however, we have attempted several selective reduction methods with no success. In all the cases tried $\left(\mathrm{NaBH}_{4}, \mathrm{LiAlH}_{4}, \mathrm{NaBH}_{3} \mathrm{CN}\right.$ etc. $)$," the unwanted reduction of vinyl group occurred.

In summary, 8-alkyl-2' (or 3')-azido(or amino)-adenosine derivatives (16-19) were successfully prepared from the 8alkylation of selectively protected adenosine derivatives $(\mathbf{1 2}$, 13) using $\operatorname{Pd}(0)$ catalyzed cross-coupling reaction with tetraalkyltin reagents, followed by the sugar modification of these 8-alkyl-adenosine derivatives according to our precedent procedure. More importantly, compared to other precedent procedures, our 8-alkylation reaction system using selectively TBDMS-protected 8-alkyl adenosine derivatives as starting materials is meaningful in terms of yie]ds and the 


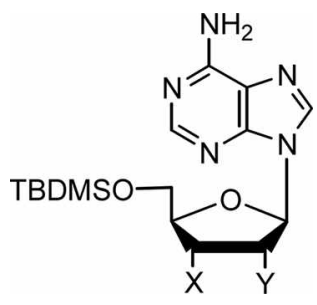

$10 \mathrm{X}=\mathrm{OTBDMS}, \mathrm{Y}=\mathrm{OH}$ $11 \mathrm{X}=\mathrm{OH}, \mathrm{Y}=\mathrm{OTBDMS}$

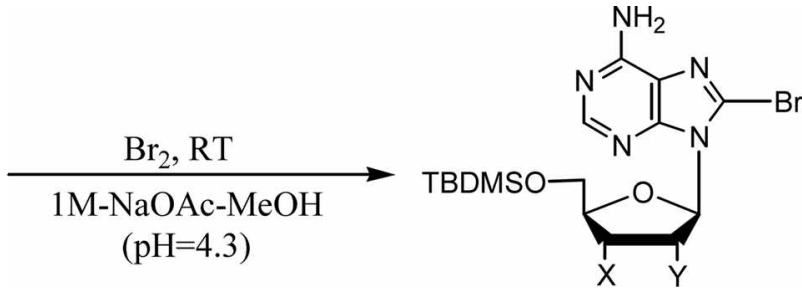

$12 \mathrm{X}=$ OTBDMS, $\mathrm{Y}=\mathrm{OH}$ $13 \mathrm{X}=\mathrm{OH}, \mathrm{Y}=\mathrm{OTBDMS}$

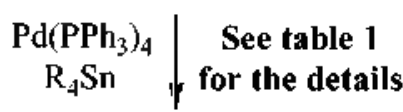<smiles>[R]c1nc2c(N)ncnc2n1[C@H]1O[C@@H](CO)[C@@H]([Y])[C@@H]1[Y]</smiles>

16a-c $X=\mathrm{OH}, \mathrm{Y}=\mathrm{N}_{3}$ 17a-c $X=\mathrm{N}_{3}, Y=\mathrm{OH}$ 18a-c $X=\mathrm{OH}, Y=\mathrm{NH}_{2}$ 19a-c $X=\mathrm{NH}_{2}, \mathrm{Y}=\mathrm{OH}$

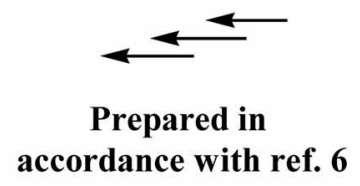

Prepared in accordance with ref. 6

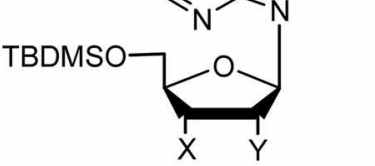

14a-c $X=$ OTBDMS, $Y=O H$

15a-c $X=O H, Y=O T B D M S$

a $\mathrm{R}=\mathrm{Me}, \mathrm{b} \mathrm{R}=\mathrm{Et}, \mathbf{c} \mathrm{R}=\mathrm{Vinyl}$

Scheme 1

Table 1.

\begin{tabular}{|c|c|c|c|c|c|}
\hline \multirow[b]{2}{*}{ Entry } & \multirow{2}{*}{$\begin{array}{l}\text { Starting } \\
\text { material }\end{array}$} & \multicolumn{3}{|c|}{ Reaction condition } & \multirow{2}{*}{$\begin{array}{l}\text { Product/ } \\
\text { yield (\%) }\end{array}$} \\
\hline & & $\begin{array}{c}\text { Pd }(0) \\
\text { catalyst }(\%)\end{array}$ & $\begin{array}{c}\mathrm{R}_{4} \mathrm{Sn} \\
\text { (equiv.) }\end{array}$ & $\begin{array}{l}\text { Reaction temp. } \\
\text { ("C)/time (h) }\end{array}$ & \\
\hline 1 & 12 & 10 & $\mathrm{R}=\mathrm{Me}(2)$ & $100 / 1$ & $14 a(90)$ \\
\hline 2 & 13 & 10 & $\mathrm{R}=\mathrm{Me}(2)$ & $100 / 2$ & $15 \mathbf{a}(92)$ \\
\hline 3 & 12 & 15 & $\mathrm{R}=\mathrm{Et}(4)$ & $100 / 1$ & $14 b(91)$ \\
\hline 4 & 13 & 12 & $\mathrm{R}=\mathrm{Et}(4)$ & $100 / 3$ & $15 \mathbf{b}(75)$ \\
\hline 5 & 12 & 15 & $\mathrm{R}=\operatorname{vinyl}(2)$ & $100 / 5$ & $14 c(67)^{4 t}$ \\
\hline 6 & 13 & 15 & $\mathrm{R}=\operatorname{vinyl}(4)$ & $100 / 1.5$ & $15 c(77)$ \\
\hline
\end{tabular}

reaction manipulation. With these important adenosine derivatives, the transfomation of them to $\mathrm{NAD}^{+}$analogues and then to cADPR is now underway.

\section{Experimental Section}

Melting points were recorded on Electrothermal melting point apparatus and are uncorrected. Mass and NMR spectra were recorded on Applied Biosystems Qstar XL and Jeo] $400 \mathrm{MHz}$ spectrometer, respectively.

A typical procedure for the 8-bromination. A solution of $10^{6}(1.3 \mathrm{~g}, 2.62 \mathrm{mmol})$ in $\mathrm{MeOH}(120 \mathrm{~mL}) / \mathrm{l} \mathrm{M}$ sodium acetate $(20 \mathrm{~mL})$ was added bromine $(0.28 \mathrm{~mL}, 5.50 \mathrm{mmol})$ at $\mathrm{rt}$ and stirred for a half-hour. The mixture was diluted with $50 \mathrm{~mL}$ of saturated sodium metabisulfite solution and stirred until the red color was disappeared. The volatile was evaporated and the resulting aqueous layer was extracted with EtOAc $(100 \mathrm{~mL} \times 3)$. The combined organic layer was washed with water $(100 \mathrm{~mL})$, dried over $\mathrm{MgSO}_{4}$, and evaporated to give pale yellowish foam. This residue was purified by column chromatography to give 12 as pale yellow solid $(1.38 \mathrm{~g}, 92 \%) .12:{ }^{1} \mathrm{H}-\mathrm{NMR}\left(\mathrm{CDCl}_{3}\right) \delta 8.13(\mathrm{~s}$, $1 \mathrm{H}, \mathrm{H} 2), 6.05(\mathrm{~s}, 1 \mathrm{H}, \mathrm{HI}), 5.89\left(\mathrm{~s}, 2 \mathrm{H}, \mathrm{NH}_{2}\right), 5.29(\mathrm{t}, 1 \mathrm{H}, J=$ $5.2 \mathrm{~Hz}, \mathrm{H} 2), 4.95$ (t, IH, $J=5.2 \mathrm{~Hz}, \mathrm{H} 3$ ), 3.95 (m, IH, H4), 3.87 (dd, 1H, $J=11.6,4.4 \mathrm{~Hz}, \mathrm{H} 5 \mathrm{a}), 3.64$ (dd, $1 \mathrm{H}, J=11.2$, $4.4 \mathrm{~Hz}, \mathrm{H} 5 \mathrm{~b}), 0.92$ (s, $9 \mathrm{H}, \mathrm{t}$-butyl), 0.71 (s, $9 \mathrm{H}, t$-butyl), 0.17 (s, 3H, methyl), 0.16 (s, 3H, methyl), -0.09 (s, 3H, methyl), $-0.19\left(\mathrm{~s}, 3 \mathrm{H}\right.$, methyl); ${ }^{13} \mathrm{C}-\mathrm{NMR} \delta 154.14,152.2 \mathrm{I}, 150.48$, $128.25,120.05,90.76,84.34,71.85,71.07,61.66,25.78$, $25.68,18.23,18.06,-4.62,-4.85,-5.52,-5.59 .13:{ }^{1} \mathrm{H}-$ $\operatorname{NMR}\left(\mathrm{CDCl}_{3}\right) \delta 8.22(\mathrm{~s}, \mathrm{IH}, \mathrm{H} 2), 5.89(\mathrm{~d}, 1 \mathrm{H}, J=5.2 \mathrm{~Hz}$, $\mathrm{H} 1), 5.74\left(\mathrm{~s}, 2 \mathrm{H}, \mathrm{NH}_{2}\right), 5.52$ (t, 1H, $\left.J=5.2 \mathrm{~Hz}, \mathrm{H} 2\right), 4.44$ (s, $1 \mathrm{H}, \mathrm{H} 3), 4.06$ (m, IH, H4), 3.97 (dd, IH, $J=11.2,6 \mathrm{~Hz}$, $\mathrm{H} 5 \mathrm{a}$ ), 3.\% (dd, $1 \mathrm{H}, J=11.2,5.2 \mathrm{~Hz}, \mathrm{H} 5 \mathrm{~b}), 2.72(\mathrm{~s}, 1 \mathrm{H}$, $-\mathrm{OH}), 0.85(\mathrm{~s}, 9 \mathrm{H}, t$-butyl $), 0.81(\mathrm{~s}, 9 \mathrm{H}, t$-butyl $), 0.01(\mathrm{~s}, 3 \mathrm{H}$, methyl), $0.00(\mathrm{~s}, 3 \mathrm{H}$, methyl), $-0.06(\mathrm{~s}, 3 \mathrm{H}$, methyl), -0.23 (s, 3H, methyl); ${ }^{13} \mathrm{C}-\mathrm{NMR} \delta 154.21,152.81,150.99,128.30$, $120.39,90.79,85.30,72.19,70.85,62.78,25.91,25.56$, $18.41,17.88,0.00,-5.00,-5.09,-5.37$.

Typical procedure of 8 -alkylated adenosine derivatives (14 and 15). To a solution of $12(0.1 \mathrm{~g}, 0.17 \mathrm{mmol})$ and tetrakis(triphenylphosphine)palladium $(0)(20 \mathrm{mg}, 0.017$ mmol) in $1 \mathrm{~mL}$ of $N$-methyl-2-pyrrolidinone (NMP) was 
added tetramethyltin $(0.05 \mathrm{~mL}, 0.35 \mathrm{mmol})$ under $\mathrm{Ar}$ atmosphere. The mixture was stirred for 1 hour at $100^{\circ} \mathrm{C}$. The mixture was partitioned between EtOAc $(50 \mathrm{~mL})$ and water $(25 \mathrm{~mL})$. The aqueous layer was extracted with EtOAc once more $(50 \mathrm{~mL})$. The combined organic layer was washed with water $(50 \mathrm{~mL} \times 2)$ and brine $(50 \mathrm{~mL})$, dried over $\mathrm{MgSO}_{4}$, evaporated to give pale yellow oily residue. The residue was applied to column chromatography $(2 \times 10$ $\mathrm{cm}$ ) and eluted with EtOAc-hexane $(7: 3)$. The appropriate fractions were collected and evaporated to give $14 \mathrm{a}(80 \mathrm{mg}$, $90 \%)$ as a white solid. 14a: 'H-NMR $\left(\mathrm{CDCl}_{3}\right) \delta 8.01(\mathrm{~s}, 1 \mathrm{H}$, $\mathrm{H} 2), 5.60\left(\mathrm{~d}, 1 \mathrm{H}, J=4.0 \mathrm{~Hz}, \mathrm{Hl}{ }^{\prime}\right), 5.26\left(\mathrm{br}, 2 \mathrm{H}, \mathrm{NH}_{2}\right), 5.02$ (t, $\left.1 \mathrm{H}, J=4.0 \mathrm{~Hz}, \mathrm{H} 2^{\prime}\right), 4.73$ (t, $1 \mathrm{H}, J=5.2 \mathrm{~Hz}, \mathrm{H}^{\prime}$ ), 3.78 (dd, $\left.1 \mathrm{H}, J=4.4,9.6 \mathrm{~Hz}, \mathrm{H} 4^{\prime}\right), 3.68(\mathrm{dd}, 1 \mathrm{H}, J=4.0,11.2 \mathrm{~Hz}$, H5'a), 3.49 (dd, 1H, $J=4.0,11.2 \mathrm{~Hz}, \mathrm{H} 5{ }^{\prime} \mathrm{b}$ ), 3.00 (br, $1 \mathrm{H}$, $\left.\mathrm{OH} 2^{\prime}\right), 2.43$ (s, 3H, 8-methyl), 0.77 (s, 9H, t-butyl), 0.55 (s, $9 \mathrm{H}, t$-butyl), $0.02(\mathrm{~s}, 3 \mathrm{H}$, methyl), $-0.22(\mathrm{~s}, 3 \mathrm{H}$, methyl), -0.25 (s, 3H, methyl), -0.35 (s, 3H, methyl); ${ }^{13} \mathrm{C}-\mathrm{NMR} \delta$ $151.80,132.06,131.97,128.47,128.35,89.12,84.42,72.33$, $71.22,62.04,25.87,25.76,18.31,18.17,14.84,-4.56$, $-4.72,-5.47,-5.53$; TOFMS: $m / z\left(\mathrm{M}^{+}+\mathrm{H}\right) 510.14 \mathrm{~b}$ : 'H$\operatorname{NMR}\left(\mathrm{CDCl}_{3}\right) \delta 7.98(\mathrm{~s}, 1 \mathrm{H}, \mathrm{H} 2), 5.7\left(\mathrm{~s}, 2 \mathrm{H}, \mathrm{NH}_{2}\right), 5.59(\mathrm{~d}$, $1 \mathrm{H}, J=4.4 \mathrm{~Hz}, \mathrm{H} 1), 5.06(\mathrm{t}, 1 \mathrm{H}, J=4.4 \mathrm{~Hz}, \mathrm{H} 2), 4.74(\mathrm{t}, 1 \mathrm{H}$, $J=5.2, \mathrm{H} 3$ ), 3.78 (t, $1 \mathrm{H}, J=4.4 \mathrm{~Hz}, \mathrm{H} 4), 3.69$ (dd, $1 \mathrm{H}, J=$ $11.2,4.4 \mathrm{~Hz}, \mathrm{H} 5 \mathrm{a}$ ), 3.49 (dd, $1 \mathrm{H}, J=11.6,4.4 \mathrm{~Hz}, \mathrm{H} 5 \mathrm{~b}$ ), 2.73 (dd, $2 \mathrm{H}, J=15.2,7.2 \mathrm{~Hz}$, ethyl), $2.4(\mathrm{~s}, 1 \mathrm{H}, \mathrm{OH}), 1.19$ (t, $3 \mathrm{H}, J=7.2 \mathrm{~Hz}$, ethyl), $0.74(\mathrm{~s}, 9 \mathrm{H}, t$-butyl), $0.56(\mathrm{~s}, 9 \mathrm{H}, t-$ butyl), $0.01(\mathrm{~s}, 3 \mathrm{H}$, methyl), $0.00(\mathrm{~s}, 3 \mathrm{H}$, methyl), $-0.25(\mathrm{~s}$, $3 \mathrm{H}$, methyl), -0.34 (s, 3H, methyl); ${ }^{13} \mathrm{C}-\mathrm{NMR} \delta 154.82$, $154.45,151.69,150.59,118.56,88.88,84.37,72.24,71.33$, $62.09,25.86,25.75,21.53,18.30,18.16,11.92,-4.57$, $-4.73,-5.47,-5.53$; TOFMS: $m / z\left(\mathrm{M}^{+}+\mathrm{H}\right) 524$. 14c: 'HNMR $\left(\mathrm{CDCl}_{3}\right) \delta 8.03(\mathrm{~s}, 1 \mathrm{H}, \mathrm{H} 2), 6.74(\mathrm{dd}, 1 \mathrm{H}, J=16.8$, $11.2 \mathrm{~Hz}, \mathrm{Ha}), 6.24(\mathrm{~d}, 1 \mathrm{H}, J=16.8 \mathrm{~Hz}, \mathrm{Hc}), 5.74(\mathrm{~d}, 1 \mathrm{H}, J=$ $4.4 \mathrm{~Hz}, \mathrm{Hl}), 5.51\left(\mathrm{~s}, 2 \mathrm{H}, \mathrm{NH}_{2}\right), 5.47$ (d, $\left.1 \mathrm{H}, J=4.4 \mathrm{~Hz}, \mathrm{Hb}\right)$, $4.89(\mathrm{~s}, 1 \mathrm{H}, \mathrm{H} 2), 4.66(\mathrm{t}, 1 \mathrm{H}, J=4.4 \mathrm{~Hz}, \mathrm{H} 3), 3.78(\mathrm{t}, 1 \mathrm{H}, J$ $=4.4 \mathrm{~Hz}, \mathrm{H} 4), 3.69(\mathrm{dd}, 1 \mathrm{H}, J=11.2,3.6 \mathrm{~Hz}, \mathrm{H} 5 \mathrm{a}), 3.52(\mathrm{dd}$, $1 \mathrm{H}, J=11.2,3.6 \mathrm{~Hz}, \mathrm{H} 5 \mathrm{~b}), 3.06(\mathrm{~s}, 1 \mathrm{H}, \mathrm{OH}), 0.76(\mathrm{~s}, 9 \mathrm{H}, t-$ butyl), 0.58 ( $\mathrm{s}, 9 \mathrm{H}, i$-butyl), $0.00(\mathrm{~s}, 3 \mathrm{H}$, methyl), 0.01 (s, $3 \mathrm{H}$, methyl), -0.23 (s, 3H, methyl), -0.32 (s, 3H, methyl); ${ }^{13}$ C-NMR $\delta 154.78,152.21,150.40,149.12,123.80,123.70$, $119.14,88.35,84.63,72.62,70.87,61.87,25.78,25.71$, $18.26,18.09,-4.63,-4.78,-5.55,-5.57$; TOFMS: $m / z$ $\left(\mathrm{M}^{+}+\mathrm{H}\right)$ 522. 15a: 'H-NMR $\left(\mathrm{CDCl}_{3}\right) \delta 8.20(\mathrm{~s}, 1 \mathrm{H}, \mathrm{H} 2)$, $5.78\left(\mathrm{~d}, 1 \mathrm{H}, J=5.6 \mathrm{~Hz}, \mathrm{H} 1^{\prime}\right), 5.78\left(\mathrm{~s}, 2 \mathrm{H}, \mathrm{NH}_{2}\right), 5.37(\mathrm{t}, 1 \mathrm{H}$, $\left.J=6.0 \mathrm{~Hz}, \mathrm{H} 2^{\prime}\right), 4.31\left(\mathrm{t}, 1 \mathrm{H}, J=2.4 \mathrm{~Hz}, \mathrm{H}^{\prime}\right), 4.06(\mathrm{~m}, 1 \mathrm{H}$, H4'), 3.94 (dd, $1 \mathrm{H}, J=11.2 \mathrm{~Hz}, 5.2 \mathrm{~Hz}, \mathrm{H} 5{ }^{\prime}$ ) $), 3.75$ (dd, $1 \mathrm{H}$, $\left.J=10.8 \mathrm{~Hz}, 4.4 \mathrm{~Hz}, \mathrm{H} 5{ }^{\prime} \mathrm{b}\right), 2.57$ (s, 3H, methyl), 0.83 (s, 9H, $t$-butyl), $0.75(\mathrm{~s}, 9 \mathrm{H}, t$-butyl), $0.00(\mathrm{~s}, 3 \mathrm{H}$, methyl), $-0.00(\mathrm{~s}$, $3 \mathrm{H}$, methyl), -0.12 (s, 3H, methyl), -0.33 (s, 3H, methyl); ${ }^{13}$ C-NMR $\delta 154.40,152.14,151.15,150.15,118.66,88.49$, $85.04,72.40,70.88,62.90,25.89,25.51,18.38,17.83$, $14.87,-5.15,-5.21,-5.42,-5.44$; TOFMS: $m / z\left(\mathrm{M}^{+}+\mathrm{H}\right)$ $510.15 \mathrm{~b}$ : 'H-NMR $\left(\mathrm{CDCl}_{3}\right) \delta 8.21(\mathrm{~s}, 1 \mathrm{H}, \mathrm{H} 2), 5.77(\mathrm{~d}, 1 \mathrm{H}$, $J=6.0 \mathrm{~Hz}, \mathrm{H1}), 5.72\left(\mathrm{~s}, 2 \mathrm{H}, \mathrm{NH}_{2}\right), 5.58(\mathrm{t}, 1 \mathrm{H}, J=6.0 \mathrm{~Hz}$, H2), 4.32 (d, $1 \mathrm{H}, J=2.4, \mathrm{H} 3$ ), 4.08 (s, 1H, H4), 3.96 (dd, $1 \mathrm{H}, J=10.8,6.0 \mathrm{~Hz}, \mathrm{H} 5 \mathrm{a}), 3.74$ (dd, $1 \mathrm{H}, J=10.8,4.8 \mathrm{~Hz}$,
$\mathrm{H} 5 \mathrm{~b}$ ), 2.88 (dd, $2 \mathrm{H}, J=7.6,3.6 \mathrm{~Hz}$, ethyl), $2.85(\mathrm{~s}, 1 \mathrm{H}, \mathrm{OH}$ ), 1.36 (t, $3 \mathrm{H}, J=7.6 \mathrm{~Hz}$, ethyl), 0.84 (s, $9 \mathrm{H}, t$-butyl), 0.76 (s, $9 \mathrm{H}, t$-butyl), $0.00(\mathrm{~s}, 6 \mathrm{H}$, dimethyl), -0.13 (s, 3H, methyl), -0.34 (s, 3H, methyl); ${ }^{13} \mathrm{C}-\mathrm{NMR} \delta 154.80,154.51,151.90$, $151.01,118.80,88.26,84.96,71.96,71.10,62.86,25.94$, $25.58,21.63,18.43,17.90,12.29,-5.04,-5.13,-5.32$; TOFMS: $m / z\left(\mathrm{M}^{+}+\mathrm{H}\right) 524.15 \mathrm{c}$ ' $\mathrm{H}-\mathrm{NMR}\left(\mathrm{CDCl}_{3}\right) \delta 8.21(\mathrm{~s}$, $1 \mathrm{H}, \mathrm{H} 2), 6.97(\mathrm{dd}, 1 \mathrm{H}, J=17.2,11.2 \mathrm{~Hz}, \mathrm{Ha}), 6.41(\mathrm{~d}, 1 \mathrm{H}, J$ $=17.2 \mathrm{~Hz}, \mathrm{Hc}), 5.98(\mathrm{~d}, 1 \mathrm{H}, J=6.4 \mathrm{~Hz}, \mathrm{H1}), 5.59(\mathrm{~d}, 1 \mathrm{H}, J=$ $11.2 \mathrm{~Hz}, \mathrm{Hb}), 5.53\left(\mathrm{~s}, 2 \mathrm{H}, \mathrm{NH}_{2}\right), 4.96(\mathrm{t}, 1 \mathrm{H}, J=6.4 \mathrm{~Hz}, \mathrm{H} 2$ ), $4.24(\mathrm{t}, 1 \mathrm{H}, J=3.2 \mathrm{~Hz}, \mathrm{H} 3), 4.05$ (d, $1 \mathrm{H}, J=3.2 \mathrm{~Hz}, \mathrm{H} 4)$, $3.92(\mathrm{dd}, 1 \mathrm{H}, J=11.2,3.2 \mathrm{~Hz}, \mathrm{H} 5 \mathrm{a}), 3.77$ (dd, $1 \mathrm{H}, J=11.2$, $3.2 \mathrm{~Hz}, \mathrm{H} 5 \mathrm{~b}), 2.76(\mathrm{~d}, 1 \mathrm{H}, \mathrm{OH}), 0.83$ (s, $9 \mathrm{H}, t$-butyl), 0.68 (s, $9 \mathrm{H}, \mathrm{t}$-butyl), 0.00 ( $\mathrm{s}, 6 \mathrm{H}$, dimethyl), -0.22 (s, 3H, methyl), -0.43 (s, 3H, methyl); ${ }^{13} \mathrm{C}-\mathrm{NMR} \delta 154.82,152.59,151.08$, $148.98,124.09,123.94,119.26,87.33,85.19,73.55,70.35$, $62.95,26.01,25.54,18.52,17.89,-5.16,-5.23,-5.28$, -5.31 ; TOFMS: $m / z\left(\mathrm{M}^{+}+\mathrm{H}\right) 522$.

The preparation of 8-Alkyl-2' (or 3')-azido (or amino)2' (or 3')-deoxyadenosine derivatives (16-19): Compounds (16-19) were prepared by applying the same procedures described in the reference 6. 16a: $\mathrm{mp} 200{ }^{\circ} \mathrm{C}$ (decomp.); ${ }^{1} \mathrm{H}$ NMR (DMSO) $\delta 8.07(\mathrm{~s}, 1 \mathrm{H}, \mathrm{H} 2), 7.26\left(\mathrm{~s}, 1 \mathrm{H}, \mathrm{NH}_{2}\right), 6.02$ $(\mathrm{d}, 1 \mathrm{H},-\mathrm{OH}), 5.91(\mathrm{~d}, 1 \mathrm{H}, J=6.8 \mathrm{~Hz}, \mathrm{Hl}), 5.45 \mathrm{dd}, 1 \mathrm{H}, J=$ $7.6,4.4 \mathrm{~Hz},-\mathrm{OH}), 4.91(\mathrm{t}, 1 \mathrm{H}, J=6.0 \mathrm{~Hz}, \mathrm{H} 2), 4.57(\mathrm{dd}, 1 \mathrm{H}$, $J=9.2,6.0 \mathrm{~Hz}, \mathrm{H} 3$ ), 3.97 (dd, $1 \mathrm{H}, J=7.2,3.2 \mathrm{~Hz}, \mathrm{H} 4), 3.34-$ $3.69(\mathrm{~m}, 2 \mathrm{H}, \mathrm{H} 5), 2.55$ (s, 3H, methyl); ${ }^{13} \mathrm{C}-\mathrm{NMR} ; \delta 155.35$, $151.70,149.88,148.63,117.85,86.21,85.80,71.13,62.61$, 61.34, 14.34; TOFMS: $m / z\left(\mathrm{M}^{+}+\mathrm{H}\right) 307.16 \mathrm{~b}: \mathrm{mp} 190{ }^{\circ} \mathrm{C}$; 'H-NMR (DMSO) $\delta 7.89(\mathrm{~s}, 1 \mathrm{H}, \mathrm{H} 2), 7.10\left(\mathrm{~s}, 2 \mathrm{H}, \mathrm{NH}_{2}\right)$, $5.85(\mathrm{~d}, 1 \mathrm{H}, \mathrm{OH}), 5.72(\mathrm{~d}, 1 \mathrm{H}, J=6.8 \mathrm{~Hz}, \mathrm{H1}), 5.30(\mathrm{dd}, 1 \mathrm{H}$, $\mathrm{OH}), 4.81(\mathrm{t}, 1 \mathrm{H}, J=6.4 \mathrm{~Hz}, \mathrm{H} 2), 4.40(\mathrm{dd}, 1 \mathrm{H}, J=8.8,5.2$ $\mathrm{Hz}, \mathrm{H} 3), 3.80$ (t, $1 \mathrm{H}, J=3.2 \mathrm{~Hz}, \mathrm{H} 4), 3.48(\mathrm{~m}, 1 \mathrm{H}, \mathrm{H5a})$, 3.35 ( $\mathrm{m}, 1 \mathrm{H}, \mathrm{H} 5 \mathrm{~b}), 2.72$ (dd, $2 \mathrm{H}, J=14.4,7.2 \mathrm{~Hz}$, ethyl), 1.13 (t, $3 \mathrm{H}, J=7.2 \mathrm{~Hz}$, ethyl); ${ }^{13} \mathrm{C}-\mathrm{NMR} \delta 155.34,152.95$, $151.56,149.75,117.92,86.32,85.64,71.21,62.42,61.50$, 20.79, 11.96; TOFMS: $\mathrm{m} / \mathrm{z}\left(\mathrm{M}^{+}+\mathrm{H}\right) 321.16 \mathrm{c}: \mathrm{mp} 190^{\circ} \mathrm{C}$ (decomp.); ${ }^{1} \mathrm{H}-\mathrm{NMR}$ (DMSO) $\delta 8.09$ (s, 1H, H2), 7.44 (s, $2 \mathrm{H}, \mathrm{NH}_{2}$ ), 7.10 (dd, $1 \mathrm{H}, J=17.2,10.8 \mathrm{~Hz}, \mathrm{Ha}$ ), 6.34 (dd, $1 \mathrm{H}, J=17.2,2.0 \mathrm{~Hz}, \mathrm{Hc}), 6.15(\mathrm{~s}, 1 \mathrm{H}, \mathrm{OH}), 5.92(\mathrm{~d}, 1 \mathrm{H}, J=$ $6.8 \mathrm{~Hz}, \mathrm{H1}), 5.78(\mathrm{~s}, 1 \mathrm{H}, \mathrm{OH}), 5.69(\mathrm{~s}, 1 \mathrm{H}, J=10.8,2 \mathrm{~Hz}$, $\mathrm{Hb}), 5.17$ (t, $1 \mathrm{H}, J=6.4 \mathrm{~Hz}, \mathrm{H} 2), 4.36$ (q, $1 \mathrm{H}, J=2.8 \mathrm{~Hz}$, $\mathrm{H} 3$ ), 3.95 (t, $1 \mathrm{H}, J=2.8 \mathrm{~Hz}, \mathrm{H} 4), 3.66$ (d, $1 \mathrm{H}, J=12.0 \mathrm{~Hz}$, H5a), 3.58 (d, $1 \mathrm{H}, J=8.0 \mathrm{~Hz}, \mathrm{H} 5 \mathrm{~b})$; ${ }^{13} \mathrm{C}-\mathrm{NMR} \delta 155.78$, $152.02,149.53,147.51,123.89,123.17,118.75,87.52$, $82.95,72.62,62.05,61.77$; TOFMS: $m / z\left(\mathrm{M}^{+}+\mathrm{H}\right) 319.17 \mathrm{a}$ : mp $178^{\circ} \mathrm{C}$; 'H-NMR (DMSO) $\delta 8.00(\mathrm{~s}, 1 \mathrm{H}, \mathrm{H} 2), 7.23(\mathrm{~s}$, $\left.2 \mathrm{H}, \mathrm{NH}_{2}\right), 6.09$ (d, $\left.1 \mathrm{H},-\mathrm{OH}\right), 5.89$ (dd, $\left.1 \mathrm{H},-\mathrm{OH}\right), 5.69$ (d, $1 \mathrm{H}, J=6.8 \mathrm{~Hz}, \mathrm{H1}$ ), 5.13 (dd, $1 \mathrm{H}, J=12.4,6 \mathrm{~Hz}, \mathrm{H} 2), 4.31$ (dd, $1 \mathrm{H}, J=5.6,2.4 \mathrm{~Hz}, \mathrm{H} 3$ ), 3.90 (dd, $1 \mathrm{H}, J=6.0,2.4 \mathrm{~Hz}$, H4), 3.59 (m, 1H, H5a), 3.28 (m, 1H, H5b), 2.49 (s, 3H, methyl); ${ }^{13} \mathrm{C}-\mathrm{NMR} \delta 155.45,151.43,149.56,148.92$, $118.04,88.38,83.15,72.47,62.42,62.00,14.30$; TOFMS: $m / z\left(\mathrm{M}^{+}+\mathrm{H}\right) 307.17 \mathrm{~b}: \mathrm{mp} 208{ }^{\circ} \mathrm{C}$ (decomp.); ${ }^{1} \mathrm{H}-\mathrm{NMR}$ (DMSO) $\delta 8.06(\mathrm{~s}, 1 \mathrm{H}, \mathrm{H} 2), 7.30\left(\mathrm{br}, 2 \mathrm{H}, \mathrm{NH}_{2}\right), 6.24(\mathrm{~d}, 1 \mathrm{H}$, $J=5.6 \mathrm{~Hz},-\mathrm{OH}), 6.02\left(\mathrm{dd}, 1 \mathrm{H}, J=3.6,9.2 \mathrm{~Hz}, \mathrm{H1}{ }^{\prime}\right), 5.74(\mathrm{~d}$, $1 \mathrm{H}, J=6.8 \mathrm{~Hz},-\mathrm{OH}), 5.26\left(\mathrm{dd}, 1 \mathrm{H}, J=6.0,12.4 \mathrm{~Hz}, \mathrm{H} 2^{\prime}\right)$, 
$4.39\left(\mathrm{dd}, 1 \mathrm{H}, J=2.8,6.0 \mathrm{~Hz}, \mathrm{H}^{\prime}\right), 3.96(\mathrm{~d}, 1 \mathrm{H}, J=2.8 \mathrm{~Hz}$, H4'), 3.66 (m, 1H, H' 'a), 3.55 (m, 1H, H5'b), 2.89 (q, 2H, $J$ $=7.6 \mathrm{~Hz}$, ethyl), 1.29 (t, $3 \mathrm{H}, J=7.6 \mathrm{~Hz}$, ethyl); ${ }^{13} \mathrm{C}-\mathrm{NMR} \delta$ $155.44,153.30,151.32,149.48,118.08,88.13,83.21,72.31$, $62.54,62.10,20.85,12.18$; TOFMS: $\mathrm{m} / \mathrm{z}\left(\mathrm{M}^{+}+\mathrm{H}\right) 321.17 \mathrm{c}:$ mp $170^{\circ} \mathrm{C}$; 'H-NMR (DMSO) $\delta 8.09(\mathrm{~s}, 1 \mathrm{H}, \mathrm{H} 2), 7.44(\mathrm{~s}$, $2 \mathrm{H}, \mathrm{NH}_{2}$ ), 7.10 (dd, $1 \mathrm{H}, J=17.2,10.8 \mathrm{~Hz}, \mathrm{Ha}$ ), 6.34 (dd, $1 \mathrm{H}, J=17.2,2 \mathrm{~Hz}, \mathrm{Hc}), 6.15(\mathrm{~s}, 1 \mathrm{H},-\mathrm{OH}), 5.92(\mathrm{~d}, 1 \mathrm{H}, J=$ $6.8 \mathrm{~Hz}, \mathrm{H} 1), 5.78(\mathrm{~s}, 1 \mathrm{H},-\mathrm{OH}), 5.69(\mathrm{~s}, 1 \mathrm{H}, J=10.8,2.0 \mathrm{~Hz}$, $\mathrm{Hb}), 5.17(\mathrm{t}, 1 \mathrm{H}, J=6.4 \mathrm{~Hz}, \mathrm{H} 2), 4.36(\mathrm{q}, 1 \mathrm{H}, J=2.8 \mathrm{~Hz}$, $\mathrm{H} 3), 3.95$ (t, $1 \mathrm{H}, J=2.8 \mathrm{~Hz}, \mathrm{H} 4), 3.66$ (d, $1 \mathrm{H}, J=12.0 \mathrm{~Hz}$, H5a), 3.58 (d, $1 \mathrm{H}, J=8.0 \mathrm{~Hz}, \mathrm{H} 5 \mathrm{~b}) ;{ }^{13} \mathrm{C}-\mathrm{NMR} \delta 155.78$, $152.02,149.53,147.51,123.89,123.17,118.75,87.52$, 82.95, 72.62, 62.05, 61.77; TOFMS: $m / z\left(\mathrm{M}^{+}+\mathrm{H}\right) 319.18 \mathrm{a}:$ mp $114{ }^{\circ} \mathrm{C} ;{ }^{1} \mathrm{H}-\mathrm{NMR}$ (DMSO) $\delta 8.06(\mathrm{~s}, 1 \mathrm{H}, \mathrm{H} 2), 5.92(\mathrm{~d}$, $1 \mathrm{H}, J=8.4 \mathrm{~Hz}, \mathrm{H1}$ ), 4.54 (dd, $1 \mathrm{H}, J=8.0,5.2 \mathrm{~Hz}, \mathrm{H} 2$ ), 4.38 (d, $1 \mathrm{H}, J=5.2 \mathrm{~Hz}, \mathrm{H3}$ ), 4.16 (d, $1 \mathrm{H}, J=1.2 \mathrm{~Hz}, \mathrm{H} 4), 3.82$ (dd, $1 \mathrm{H}, J=12.8,2.8, \mathrm{H} 5 \mathrm{a}$ ), 3.69 (dd, $1 \mathrm{H}, J=12.8,2.4 \mathrm{~Hz}$, H5b), 3.14 (s, 3H, methyl); ${ }^{13} \mathrm{C}-\mathrm{NMR} \delta 156.51,152.49$, $151.85,150.88,119.45,89.80,89.56,72.76,63.89,56.73$, 14.57; TOFMS: $m / z\left(\mathrm{M}^{+}+\mathrm{H}\right) 281.18 \mathrm{~b}: \mathrm{mp} 188^{\circ} \mathrm{C} ;{ }^{1} \mathrm{H}-\mathrm{NMR}$ (DMSO) $\delta 8.09(\mathrm{~s}, 1 \mathrm{H}, \mathrm{H} 2), 7.29\left(\mathrm{~s}, 2 \mathrm{H}, \mathrm{NH}_{2}\right), 6.15(\mathrm{~s}, 1 \mathrm{H}$, $-\mathrm{OH}), 5.62(\mathrm{~d}, 1 \mathrm{H}, J=8.0 \mathrm{~Hz}, \mathrm{H1}), 4.52(\mathrm{~s}, 1 \mathrm{H},-\mathrm{OH}), 4.24$ (d, $1 \mathrm{H}, J=8.0 \mathrm{~Hz}, \mathrm{H} 2), 4.08$ (d, $2 \mathrm{H}, \mathrm{H} 3,4), 3.73-3.61$ (m, $2 \mathrm{H}, \mathrm{H} 5 \mathrm{a}, \mathrm{b}), 2.96$ (d, $2 \mathrm{H}, J=7.2 \mathrm{~Hz}$, ethyl), 1.37 (t, $3 \mathrm{H}, J=$ $7.2 \mathrm{~Hz}$, ethyl); ${ }^{13} \mathrm{C}-\mathrm{NMR} \delta 155.36,153.45,150.90,149.42$, $118.15,89.50,87.42,71.97,62.74,56.04,20.88,12.14$; TOFMS: $m / z\left(\mathrm{M}^{+}+\mathrm{H}\right)$ 295. 19a: $\mathrm{mp} 136{ }^{\circ} \mathrm{C}$; ${ }^{1} \mathrm{H}-\mathrm{NMR}$ (DMSO) $\delta 8.00(\mathrm{~s}, 1 \mathrm{H}, \mathrm{H} 2), 7.16\left(\mathrm{~s}, 2 \mathrm{H}, \mathrm{NH}_{2}\right), 5.79(\mathrm{~d}, 1 \mathrm{H}$, $J=5.2 \mathrm{~Hz}, \mathrm{H1}), 4.71(\mathrm{t}, 1 \mathrm{H}, J=5.6 \mathrm{~Hz}, \mathrm{H} 2), 3.78(\mathrm{~s}, 1 \mathrm{H}$, $-\mathrm{OH}), 3.65(\mathrm{~d}, 1 \mathrm{H}, J=12 \mathrm{~Hz}, \mathrm{H} 3), 3.58(\mathrm{t}, 1 \mathrm{H}, J=5.2 \mathrm{~Hz}$, H4), 3.46 (dd, $2 \mathrm{H}, J=2.8,12.4 \mathrm{~Hz}, \mathrm{H} 5$ ), 2.49 (s, 3H, methyl); ${ }^{13} \mathrm{C}-\mathrm{NMR} \quad \delta 155.18,151.30,149.58,148.59$, $117.85,89.46,85.37,72.07,62.14,53.08,14.43$; TOFMS: $m / z\left(\mathrm{M}^{+}+\mathrm{H}\right) 281.19 \mathrm{~b}: \mathrm{mp} 195^{\circ} \mathrm{C} ;{ }^{1} \mathrm{H}-\mathrm{NMR}$ (DMSO) $\delta 7.80$ (s, $1 \mathrm{H}, \mathrm{H} 2), 5.80(\mathrm{~d}, 1 \mathrm{H}, J=5.2 \mathrm{~Hz}, \mathrm{H} 1), 4.82(\mathrm{t}, 1 \mathrm{H}, \mathrm{H} 2)$, $3.77-3.59(\mathrm{~m}, 4 \mathrm{H}, \mathrm{H} 3,4,5 \mathrm{a}, \mathrm{b}), 2.71(\mathrm{~d}, 2 \mathrm{H}, J=8.4 \mathrm{~Hz}$, ethyl), 1.12 (t, $3 \mathrm{H}, J=7.6 \mathrm{~Hz}$, ethyl); ${ }^{13} \mathrm{C}-\mathrm{NMR} \delta 156.22$, $155.76,152.14,151.85,117.87,89.79,84.71,72.36,60.99$, $52.96,21.58,11.62$; TOFMS: $m / z\left(\mathrm{M}^{+}+\mathrm{H}\right) 295$.

Acknowledgements. This work was supported from Korean Research Foundation Grant (KRF-2004-005-F00015) endowed to RCBM of Chonbuk National University in 2005.

\section{References}

I. 1. (a) Lee, H. C.; Munshi, C.; Graeff, R. Mol. Cell Biochent 1999, 193, 89. (b) Takasawa, S.; Nata, S.; Yonekura, H.; Okamoto, H. Science 1993, 259, 370. (c) Lee, H. C. Mol. \& Cell Biochem. 1994, 138, 229. (d) Currie, K.; Swann, K.; Galione, A.; Scott, R. H. Mol. Biol. Cell 1992, 3, 1415 .

2. (a) Takasawa, S.; Akiyama, T.; Nata, K.; Kuroki, M.; Tohgo, A.; Noguchi. N.; Kobayashi. S.; Kato, I.; Katada, T.; Okamoto, H. $J$. Biol. Chem, 1998, 273, 2497. (b) Takasawa, S.; Nata, K.; Yonekura, H.; Okamolo, H. Science 1993, 259, 370. (c) Javaraman, T.; Ondriasova, E.; Ondrias, K.; Harnick, D. J.; Marks, A. R. Proc. Notl. Acad. Sci. 1995, 92, 6007. (d) Guse, A. H.; da Silva, C. P; Berg. I.; Weber, K.; Heyer, P.; Hohenegger, M.; Ashamu, G A.; Skapenko, A. L.; Schulze-Koops, H.; Potter, B. V. L.; Mavr, G. W. Nature 1999, 398, 70. (e) Rakovic, R.; Cui, Y.; Jino, S.; Galione, A.; Ashamu, G. A.; Potter, B. V. L.; Terrar, D. A. J. Biol. Chem. 1999, 274, 17820. (I) Guse, A. H. Curr. Mol. Med. 2004, 4, 239.

3. (a) Lee, H. C. Cttrr Mol. Med. 2004, 4, 227. (b) Potter, B. V. L.; Walseth, T. F. Ctrrr. Mol. Med. 2004, 4, 308. (c) Shuto, S.; Matsuda A. Curr. Mol. Med. 2004. H, 827. (d) Guse. A. H. J. Mol, Med. 2000, 78, 26. (e) Zhang, F.-J.; Gu, Q.-M.; Sih, C. J. Bioorg. \& Med. Chem. 1999, 7,653.

4. (a) Guse, A. H.; da Silva, C. P.; Emmrich, F.; Ashamu, G. A.; Potter, B. V. L.; Mayr, G. W. J. Immunol. 1995, 155, 353. (b) Guse, A. H.; da Silva, C. P.; Weber, K.; Armah, C.; Schulze, C.; Potter, B. V. L.; Mayr, G. W.; Hilz, H. Eur. J. Biochem. 1997, 245, 411 .

5. (a) Bailey, V. C.; Sethi, J. K.; Forth, \$. M.; Galione, A.; Potter, B. V. L. Chemistry \& Biology 1997, 4, 5I. (b) Shuto, S.; Fukuoka, M.; Manikowsky, A.; Ueno, Y.; Nakano, T.; Kuroda, H.; Kuroda, H.; Matsuda, A. J. Aml. Chem. Soc. 2001, 233.8750 . (c) Wong. L.; Aarhus, R.; Lee, H. C.; Walseth, T. F. Biochim. Biophys. Acta 1999, 1472,555 .

6. Kim, B.-T.; Kim, S.-K.; Lee, S.-J.; Hwang, K.-J. Bull. Korean Chem. Soc. 2004, 25, 243.

7. (a) Ueda, T.; Matsuda, A. Chem. Pham. Bull, 1984, 33, 3236. (b) Matsuda, A.; Nomoto, Y.; Ueda, T. Chem. Pharm. Bull. 1979. 27, 183. (c) Sarfati, S. R.; Pochet, S.; Guerteiro, C.; Namane, A.; Huynh-Dinh, T.; Igolen, J. Tetrahedron 1987, 43, 3491.

8. (a) Hayakawa, H.; Haraguchi, K.; Tanaka, H.; Miyasaka, T. Chem. Pharm, Bull. 1987, 35, 72, (b) Barton, D. H. R.; Hedgecock, C. J. R.; Lederer, E.; Motherwell, W. B. Tetrahedron Lett, 1979, 279.

9. (a) Nair. V.; Purdy, D. F. Tetrahedron 1991. 47. 365. (b) Moriarty, R. M.; Epa, W. R.; Awasthi, A. K. Tetrahedron Lett. 1990, 31, 5877. (c) Kitade, Y.; Nakata, Y;; Hirota, K.; Maki, Y.; Pabuccuoglu, A.; Tortence, P. F. Nucleic Acids Res, 1991, 19, 4103. (d) Van Aerschol, A. A.; Mamos, P.; Weyns, N. J.; Ikeda, S.; De Clercq, E.; Hedewijn. P. A. J. Med. Chen. 1993, 36, 2938. (e) Hirota. K.; Kitade, Y.; Kanbe, Y.; Maki, Y. J. Org. Chent. 1992, 57, 5268.

10. Zhang. W. Robins, M. J. Tetrahedron Lett. 1992, 33, 1177.

11. (a) Spurlock, L. A.; Schultz, R. J. J. Am. Chem. Sac. 1970, 92 , 6302. (b) Spurlock, L. A.; Cox, W. G. J. Am. Chem. Soc. 1969, 91 , 2961 . 